\title{
Second-Line Antiretroviral Therapy in Sub-Saharan Africa: It Is Time to Mind the Gaps
}

\author{
Richard A. Murphy, Richard Court, Gary Maartens, ${ }^{2}$ and Henry Sunpath ${ }^{3}$
}

\begin{abstract}
The delay between first-line antiretroviral therapy (ART) failure and initiation of second-line ART in resource-limited settings can be prolonged. Increasing evidence links delayed antiretroviral switch with increased risk for opportunistic infection (OI) and death, particularly in patients with advanced HIV at the time of first-line failure. As access to viral load (VL) monitoring widens beyond a few countries, mechanisms are needed to optimize the use of routine virologic monitoring and assure that first-line regimen failure results in prompt second-line switch. For patients with advanced HIV or OI at the time of first-line failure, a targeted fast track to second-line ART should be considered, involving a switch to second-line ART during a single visit. To derive the maximum benefit from both the current expansion of VL monitoring and the falling costs of second-line ART, clinics and healthcare workers should be given the tools and training to detect and switch patients with regimen failure before HIV disease progression.
\end{abstract}

Keywords: second-line antiretroviral therapy, antiretroviral failure, developing world, sub-Saharan Africa, virologic monitoring, opportunistic infection

\section{Introduction}

A LTHOUGH MILLIONS IN sub-Saharan Africa have initiated antiretroviral therapy (ART), which has resulted in steep declines in HIV-related deaths, the number of patients experiencing first-line ART failure, who have required second-line protease inhibitor-based therapy, is growing. ${ }^{1}$ In contrast to resource-rich settings, the delay between first-line ART failure and the initiation of second-line ART can be prolonged in resource-limited settings. Delayed second-line switch has now been linked in three sub-Saharan African countries with an increased risk of opportunistic infection (OI) and death. ${ }^{2-4}$

\section{The Causes and Costs of Late or Missed Second-Line Switch}

Several factors contribute to the gap between recognition of treatment failure and second-line ART switch. These factors include clinician concerns about adherence to second-line ART and toxicity of a less familiar drug regimen, laboratory infrastructure limitations, and protocolized requirements in some countries for several repeat patient clinic visits before switch. For example, national treatment guidelines in South
Africa recommend a routine 2-month delay for patients with virologic failure before initiation of second-line ART to accommodate adherence support and repeat laboratory confirmation of virologic failure. However, on the ground, switch delays in South Africa are nearly twice that-a median of 3.4 months-with $37 \%$ of patients with first-line ART failure in South Africa failing to switch. ${ }^{4}$

Studies in multiple resource-limited settings have now confirmed an increased risk for OI and mortality associated with delayed or missed second-line switch, even in settings with access to viral load (VL) monitoring, which in principle allows for prompt detection of failure. ${ }^{2-6}$ (Table 1). The conclusions of these studies are plausible given that many patients receiving first-line ART in resource-limited settings have clinically advanced HIV at the time of initial regimen failure and experience viral rebound in a context wherein the risk of OI such as tuberculosis is high.

\section{Existing Barriers to Prompt Recognition and Response to Regimen Failure}

Access to VL monitoring is likely to increase in subSaharan Africa in the next decade owing to developments in

\footnotetext{
${ }^{1}$ Division of Infectious Diseases, Los Angeles Biomedical Research Institute, Harbor-UCLA Medical Center, Torrance, California.

${ }^{2}$ Division of Clinical Pharmacology, Department of Medicine, University of Cape Town, Cape Town, South Africa.

${ }^{3}$ Division of Infectious Diseases, University of KwaZulu-Natal, Durban, South Africa.

(C) Richard A. Murphy et al. 2017; Published by Mary Ann Liebert, Inc. This article is available under the Creative Commons License CC-BY-NC (http://creativecommons.org/licenses/by-nc/4.0). This license permits non-commercial use, distribution and reproduction in any medium, provided the original work is properly cited. Permission only needs to be obtained for commercial use and can be done via RightsLink.
} 


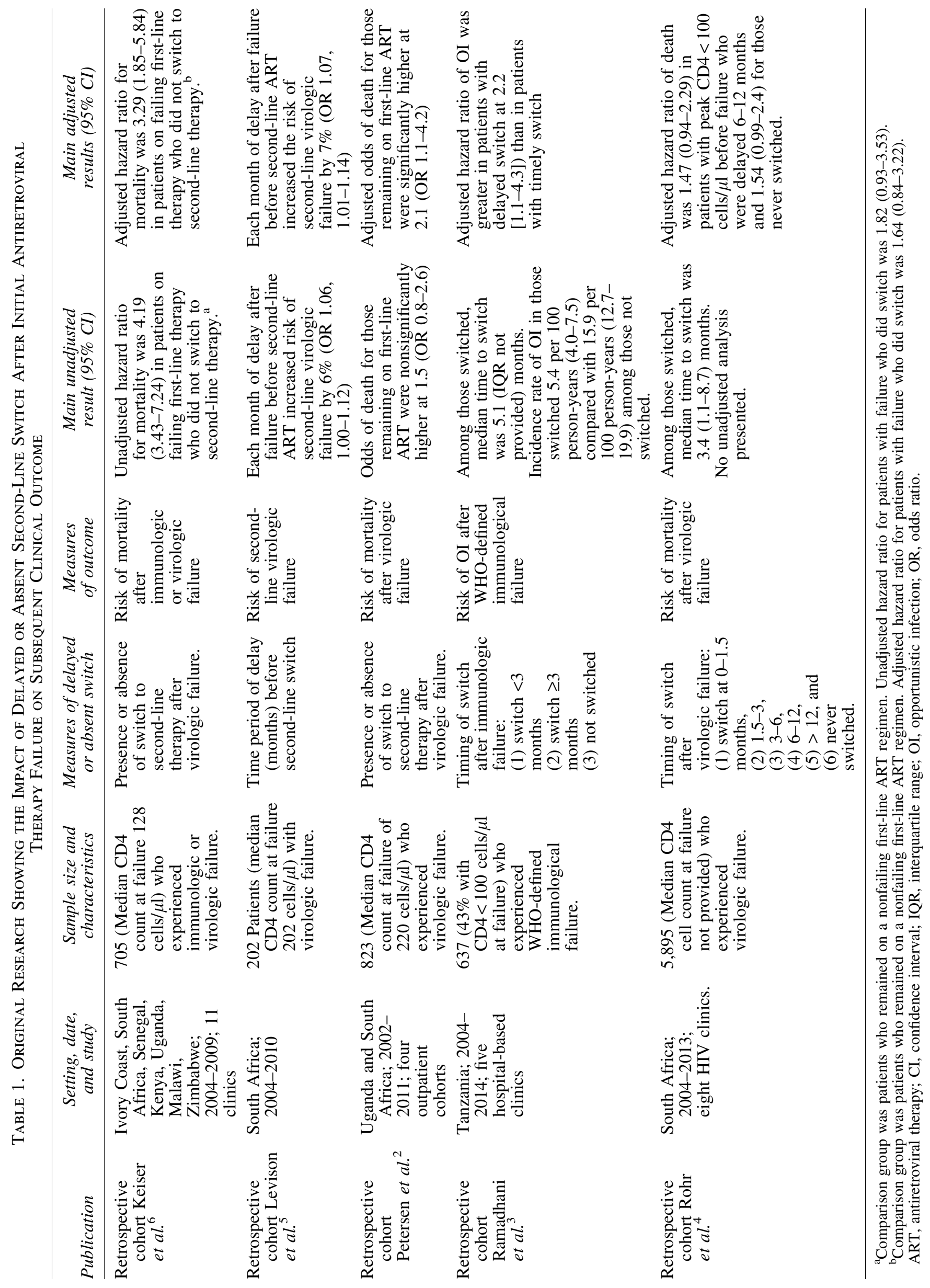


the field of diagnostics and a wider recognition of the benefits at the country level. Improving the early detection of ART failure is critical, and expanding the use of VL monitoring beyond the few countries that currently have access to it is key to achieving this. Unfortunately, evidence from South Africa strongly suggests that merely making VL routinely available in clinics is not sufficient.

In South Africa-where virologic monitoring has been in place for more than a decade-there is strong evidence that it is underutilized. Investigators in Johannesburg, seeking to compare public and private sector ART outcomes, found that in the first year after commencing ART, only $45 \%(2,938 / 6,528)$ of public sector patients and $55 \%$ $(135 / 245)$ in the private sector had a VL measured. ${ }^{7}$ Similarly, in public ART programs in KwaZulu-Natal, the proportion with a VL measured 6 months after initiating ART - a key treatment juncture-was $<50 \%$. $^{7}$ The underutilization of VL monitoring in routine HIV care represents a missed opportunity to identify and address treatment failure both for individual benefit and to limit potential transmission of drug-resistant virus in the community. ${ }^{8}$ Currently, the barriers to optimize use of VL monitoring in South Africa at the patient and clinic level are significant and include a inadequate knowledge of the role of VL monitoring in high quality care, a lack of "ownership" of VL results in clinics, a poor interface between clinics and laboratories in sample collection and results reporting, and, in some clinics, large patient volumes with insufficient human resources. However, some innovative recent approaches to reducing missed virologic monitoring opportunities in one South African municipality include: (1) identifying a clinician in each clinic to be a VL "champion" tasked with improving compliance with VL monitoring and assuring that those with persistently elevated VL are switched promptly, (2) creating an expectation among patients for yearly VL testing that includes mass media campaigns, and (3) debuting new tools to track compliance with VL monitoring standards and virologic suppression rates at the level of individual clinics to identify sites of excellence as well as outliers requiring more support.

\section{A Targeted Fast Track to Second-Line ART?}

Guidelines governing the use of second-line ART in resource-limited settings were developed when switching patients to second-line ART increased yearly per patient treatment cost by up to 17-fold. However, the cost of generic lopinavir/ritonavir $200 / 50 \mathrm{mg}$ tablets has fallen to $<\$ 250 /$ year. $^{9}$ Despite these remarkable cost improvements, second-line algorithms have not changed in many countries. To improve second-line ART access and reduce consequences from switch delay, a fast track to secondline ART for patients with regimen failure should be strongly considered. A fast track could be targeted at patients more likely to have poor outcomes resulting from delayed or missed switch, such as patients with a CD4 cell count $<200$ cells $/ \mu 1$ or OI at the time of failure while remaining vigilant of potential drug-drug interactions with a ritonavir-boosted regimen. For patients who are fast tracked, adherence support would take place after secondline switch so that such patients could benefit from adherence interventions but without, in the interim, risking disease progression or death.

The visibility of second-line switch delay could be enhanced by incorporating new monitoring indicators to assess whether patients with first-line ART failure are benefitting from second-line switch. An example of such a new indicator "Days to Second-Line Switch" would be defined, among patients with first-line ART failure, as the median days between first-line ART failure and second-line ART initiation. The goal for programs with virologic monitoring based on current evidence would be $<90$ days, particularly for patients with a CD4 cell count $<200$ cells $/ \mu 1$. Such an indicator could increase awareness of inadequate second-line ART switching and allow individual clinics or programs to be targeted for interventions to improve second-line access.

\section{Conclusions}

The consequences of delayed or missed second-line switch are becoming clear. Fortunately, the cost of secondline ART is no longer out of reach, and older approaches to switch that may have been partly premised on limiting access and delaying movement to second-line ART are no longer tenable. As access to VL monitoring improves, efforts at the implementation of VL monitoring at country level must go hand-in-hand with efforts at the clinic level to assure high VL monitoring rates and at the prescriber level, to assure that patients with virologic failure are transitioned without more than a brief delay to second-line ART. Ready access to expert advice for special situations, including HIV/OI coinfection and drug intolerance, will help reduce switch hesitancy. It is no longer a "badge of honor" for an ART program to have very few patients receiving secondline ART but rather an indication that virologic failure is going undetected and unaddressed. It is time to improve access to second-line ART, prioritizing patients who need it most urgently.

\section{Author Disclosure Statement}

No competing financial interests exist.

\section{References}

1. Estill J, Ford N, Salazar-Vizcaya L, et al.: The need for second-line antiretroviral therapy in adults in sub-Saharan Africa up to 2030: A mathematical modelling study. Lancet HIV 2016;3:e132-e139.

2. Petersen ML, Tran L, Geng EH, et al:: Delayed switch of antiretroviral therapy after virologic failure associated with elevated mortality among HIV-infected adults in Africa. AIDS 2014;28:2097-2107.

3. Ramadhani HO, Bartlett JA, Thielman NM, et al.: The effect of switching to second-line antiretroviral therapy on the risk of opportunistic infections among patients infected with human immunodeficiency virus in Northern Tanzania. Open Forum Infect Dis 2016;3:ofw018.

4. Rohr JK, Ive $\mathrm{P}$, Horsburgh CR, et al:: Marginal structural models to assess delays in second-line HIV treatment initiation in South Africa. PLoS One 2016;11: e0161469.

5. Levison JH, Orrell C, Losina E, Lu Z, Freedberg KA, Wood $\mathrm{R}$ : Early outcomes and the virologic impact of delayed 
treatment switching on second-line therapy in an antiretroviral roll-out program in South Africa. Antivir Ther 2011;16:853-861.

6. Keiser $\mathrm{O}$, Tweya $\mathrm{H}$, Braitstein $\mathrm{P}$, et al: : Mortality after failure of antiretroviral therapy in sub-Saharan Africa. Trop Med Int Heal 2010;15:251-258.

7. Moyo F, Chasela C, Brennan AT, et al:: Treatment outcomes of HIV-positive patients on first-line antiretroviral therapy in private versus public HIV clinics in Johannesburg, South Africa. Clin Epidemiol 2016;8: 37-47.

8. Marconi VC, Sunpath H, Lu Z, et al.: Prevalence of HIV-1 drug resistance after failure of a first highly active antiretroviral therapy regimen in KwaZulu Natal, South Africa. Clin Infect Dis 2008;46:1589-1597.
9. Anonymous: Untangling the web of antiretroviral price reductions. Available at www.msfaccess.org/content/untanglingweb-antiretroviral-price-reductions (2016), accessed April 1, 2017.

Address correspondence to: Richard A. Murphy, MD, MPH

Division of Infectious Diseases Los Angeles Biomedical Research Institute Harbor-UCLA Medical Center 1000 West Carson Street, Box 466 Torrance, California 90509

E-mail: ramurphy@gmail.com 